\title{
AM I SeEING Double? Absolute Configuration AND X-RAY CRYSTALlography
}

Absolute configuration plays a central role in the function and properties of many chemical compounds, from natural products to catalysts to pharmaceuticals. With a simple change of hand to a single stereocenter, carvone changes from a spearmint scent to caraway. Similarly, chiral pharmaceuticals can be active in one form, inactive, or even toxic in the other. X-ray crystallography remains the definitive method to determine absolute configuration. With large active-area CMOS detectors, bright microfocus sources, and continuous scanning modes, all of which give rise to the ability to measure only slight anomalous differences in the Friedel pairs, accurate preliminary determination of absolute configuration can be performed in just minutes, even on light-atom structures. Applications of these methods and tools against a panel of chiral compounds will be presented to demonstrate their functionality and utility.

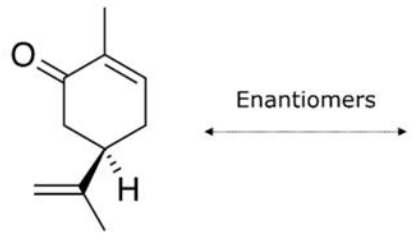

(R)-( - ) Carvone

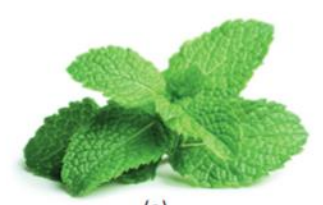

(a)

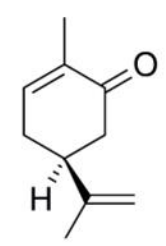

(S)-( + ) Carvone

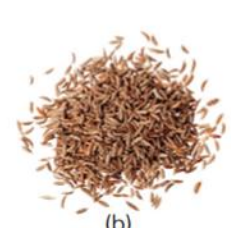

$\begin{array}{lll}\text { Bruce C. Noll } & \text { USA } & \text { Bruker AXS Inc. } \\ \text { HolgerOtt } & \text { Germany } & \text { Bruker AXS GmbH } \\ \text { Michael Ruf } & \text { USA } & \text { Bruker AXS Inc. } \\ \text { Tobias Stuerzer } & \text { Germany } & \text { Bruker AXS GmbH }\end{array}$

